\title{
Association between spicy food consumption and lipid profiles in adults: a nationwide population-based study
}

\author{
Yong Xue ${ }^{1,2} \dagger$, Tingchao $\mathrm{He}^{2} \dagger$, Kai $\mathrm{Yu}^{2}$, Ai Zhao ${ }^{3}$, Wei Zheng ${ }^{3}$, Yumei Zhang ${ }^{2 *}$ and Baoli Zhu* \\ ${ }^{1}$ Chinese Academy of Sciences (CAS) Key Laboratory of Pathogenic Microbiology and Immunology, Institute of Microbiology, \\ Chinese Academy of Science, Chaoyang District, Beijing 100101, People's Republic of China \\ ${ }^{2}$ Department of Nutrition \& Food Hygiene, School of Public Health, Peking University Health Science Center, Haidian District, \\ Beijing 100191, People's Republic of China \\ ${ }^{3}$ Department of Social Medicine E Health Education, School of Public Health, Peking University Health Science Center, \\ Haidian District, Beijing 100191, People's Republic of China
}

(Submitted 11 January 2017 - Final revision received 13 April 2017 - Accepted 17 May 2017 - First published online 4 July 2017)

\section{Abstract}

CVD remains the leading cause of mortality worldwide, with abnormal lipid metabolism as a major risk factor. The aim of this study was to investigate associations between spicy food consumption and serum lipids in Chinese adults. Data were extracted from the 2009 phase of the China Health and Nutrition Survey, consisting of 6774 apparently healthy Chinese adults aged 18-65 years. The frequency of consumption and degree of pungency of spicy food were self-reported, and regular spicy food consumption was assessed using three consecutive 24-h recalls. Total cholesterol, TAG, LDL-cholesterol and HDL-cholesterol in fasting serum were measured. Multilevel mixed-effects models were constructed to estimate associations between spicy food consumption and serum lipid profiles. The results showed that the frequency and the average amount of spicy food intake were both inversely associated with LDL-cholesterol and LDL-cholesterol:HDL-cholesterol ratio (all $P_{\text {for trend }}<0.05$ ) after adjustment for potential confounders and cluster effects. HDL-cholesterol in participants who usually consumed spicy food ( $\geq 5$ times/week) and who consumed spicy food perceived as moderate in pungency were significantly higher than those who did not (both $P<0.01$ ). The frequency and the average amount of spicy food intake and the degree of pungency in spicy food were positively associated with TAG (all $P_{\text {for trend }}<0.05$ ). Spicy food consumption was inversely associated with serum cholesterol and positively associated with serum TAG, and additional studies are needed to confirm the findings as well as to elucidate the potential roles of spicy food consumption in lipid metabolism.

Key words: Spicy foods: Serum lipids: Dyslipidaemia: CVD: China Health and Nutrition Survey

CVD, one of the largest epidemics the world has faced, accounts for more than half of all non-communicable diseases and has become the leading cause of death worldwide ${ }^{(1)}$. Dyslipidaemia is one of the major risk factors in the pathogenesis of $\mathrm{CVD}^{(2)}$, often including hypercholesteraemia, hypertriacylglycerolaemia, high LDL-cholesterol and low HDL-cholesterol. Different from those in the USA and Europe, the main types of dyslipidaemia in Asian countries including China manifest as hypertriacylglycerolaemia and low HDL-cholesterol ${ }^{(3,4)}$. A report of the China National Diabetes and Metabolic Disorders Study from 2007-2008 estimated that $9.0 \%$ ( 88.1 million) of Chinese adults had high total cholesterol (TC), 6.5\% (62.8 million) had high LDL-cholesterol and $22.3 \%$ (214.9 million) had low HDL-cholesterol ${ }^{(5)}$. With rapid socioeconomic development and resulting lifestyle changes, the prevalence of dyslipidaemia has increased dramatically over the past decade in China. Without effective intervention, it may result in the soaring prevalence of related CVD and a huge medical burden in the near future ${ }^{(5,6)}$. Healthy lifestyles, especially an appropriate dietary pattern, are key interventional targets in primordial prevention of dyslipidaemia and CVD, and lifestyle modification is recommended as part of dyslipidaemia control and management ${ }^{(7)}$. Previous research studies have indicated that some foods and their bioactive ingredients are effective in improving an individual's dyslipidaemia status ${ }^{(8-10)}$.

Capsicum species have been commonly recognised and consumed in fresh or cooked form and used as natural food colourants or spices as well as in traditional medicine worldwide as they contain many bioactive components such as small organic acids, trigonelline, choline, cinnamic derivatives or capsaicinoids $^{(11-13)}$. In China, chillies are among the most

\footnotetext{
Abbreviations: CHNS, China Health and Nutrition Survey; TC, total cholesterol.
}

* Corresponding authors: B. Zhu, fax +86 10 64807433, email zhubaoli@im.ac.cn; Y. Zhang, fax +86 1082801519 , email zhangyumei66141@163.com

$\dagger$ These authors contributed equally to this work. 
popular spicy foods consumed nationwide ${ }^{(14,15)}$. The hypolipidaemic effect of capsicum species and their bioactive ingredients was reported in animal experiments or small-sized population studies. For example, the administration of green-pepper juice was found to decrease serum TC, TAG, LDL-cholesterol and alanine aminotransferase in high-fat diet induced mice ${ }^{(16)}$. Another animal study showed that capsaicinoids, a group of pungent compounds in chillies, were beneficial in improving the lipoprotein profile and aortic function in hamsters fed a highcholesterol diet ${ }^{(17)}$, which was in agreement with a series of animal studies ${ }^{(18-22)}$. Moreover, the ingestion of chilli powder was found to decrease fasting serum TC and TAG in pregnant women with gestational diabetes mellitus (GDM) and might contribute to pregnancy outcomes (forty-two participants) ${ }^{(23)}$. In contrast, one human intervention (twenty-seven participants) reported that regular consumption of freshly chopped chillies for 4 weeks had no effect on serum lipids and lipoproteins ${ }^{(24)}$. Furthermore, another randomised crossover dietary intervention study (thirty-six participants) found no obvious beneficial or harmful effects on metabolic parameters after 4 weeks of regular chilli consumption $^{(25)}$.

These above-mentioned data suggest that spicy food may have a beneficial influence on lipid metabolism in animal models; however, the lines of evidence involving the associations between spicy food and lipid levels from human studies are inconsistent, and there have been no large populationbased cohort studies. Moreover, there has been no large population-based cohort study on the relationship between spicy food consumption and blood lipid profiles. We therefore evaluated associations between regular consumption of spicy food (frequency and the average amount of intake) and serum lipids in 6774 apparently healthy Chinese adults from the China Health and Nutrition Survey (CHNS).

\section{Methods}

\section{Study population}

Data were extracted from the CHNS, which is an ongoing longitudinal, household-based survey in nine geographically diverse areas across China with the goal to examine the nutrition and health transition of the Chinese population in the context of fast socioeconomic changes ${ }^{(26-28)}$. The CHNS was conducted in nine rounds between 1989 and 2015, and this paper used the cross-sectional data collected from the 2009 phase of the CHNS. A multistage, random cluster sampling was implemented in the CHNS, and the details of the participant-recruitment strategies have been described elsewhere ${ }^{(26,27)}$. The study sample was drawn from nine provinces including Liaoning, Heilongiang, Jiangsu, Shandong, Henan, Hubei, Hunan, Guangxi and Guizhou provinces, with a total coverage of seventy-three urban and suburban neighbourhoods within the cities and 145 villages and townships within the counties in 2009. In the present study, our analysis focused on the adult population aged 18-65 years. We excluded 1235 adult participants who had not undergone plasma lipid testing, ninety-nine with questionable lipid values, ninety-six without dietary assessment records, sixty-one pregnant women as well as six without a definite response for the frequency of spicy

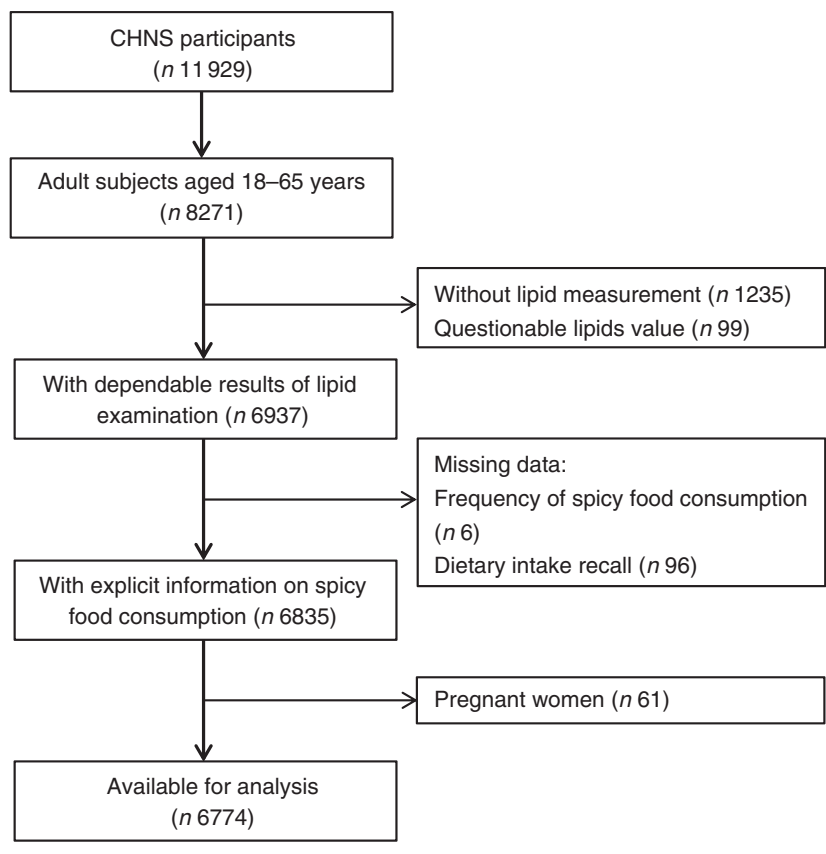

Fig. 1. Participant flow diagram. CHNS, China Health and Nutrition Survey.

food consumption, which resulted in 6774 participants (3184 men and 3590 women) in the final analysis (Fig. 1). This study was conducted according to the guidelines laid down in the Declaration of Helsinki and the research protocol was reviewed and approved by the Institute Review Board of the University of North Carolina at Chapel Hill and the National Institute of Nutrition and Food Safety, China Center for Disease Control and Prevention. All participants provided written informed consent before participation in the study.

\section{Assessment of spicy food consumption and dietary intake}

The assessment of dietary intake was based on three consecutive 24-h recalls (2 weekdays and 1 weekend day) at the individual level, which was optimised by a food inventory taken at the household level over the same $3 \mathrm{~d}^{(29,30)}$. All foods remaining after the last meal before the beginning of one 24-h dietary assessment (F1), all purchases and home production (F2) as well as food inventory (F3) at the end of one 24-h dietary assessment were weighted and recorded each day. Digital diet and kitchen scales with a minimum limit of $1 \mathrm{~g}$ and a maximum of $3 \mathrm{~kg}$ were used throughout the assessment of household consumption ${ }^{(27)}$. Preparation waste was estimated when weighing was not possible (F4), and actual household food consumption on each day was calculated as follows: $(\mathrm{F} 1+\mathrm{F} 2)-(\mathrm{F} 3+\mathrm{F} 4)$. To collect individual dietary information, trained interviewers recorded all types of food and the proportion of each dish consumed away from and at home on a 24-h-recall day with the aid of food models and pictures. The proportion of individual food consumed was estimated as a percentage of total food consumed by the household ${ }^{(27)}$. The average intakes of the three consecutive 24-h recalls were used for each individual. According to the food group classification of the Chinese Food Composition Tables from 2002 and $2004^{(31,32)}$, the average vegetable intake $(\mathrm{g} / \mathrm{d})$ of 
each individual was estimated on the basis of 24-h dietary recall as well as the average amount of spicy food (including fresh chillies, dried chillies, chilli powder, chilli sauce and chilli oil) intake. The average salt intake $(\mathrm{g} / \mathrm{d})$ of each individual was estimated on the basis of weighing the salt inventory at the household level. Total energy, carbohydrate, fat and cholesterol intakes were calculated by CHNS on the basis of the Chinese Food Composition Table and were categorised into four groups according to their quartiles as well as average vegetable intake. The average amount of spicy food intake was categorised into four predefined groups to reflect none, low, medium and high intakes. Previous studies have indicated that the combination of a consecutive 3-d 24-h recall and the household food inventory can improve the accuracy of recalls, which may contribute to the assessment of the subgroups of food and nutrient intakes ${ }^{(15,27,33)}$.

In the 2009 survey, participants were asked 'Do you like to eat hot pepper or spicy food?', with five response categories: (1) no, (2) sometimes ( $\leq 2$ times/week), (3) often (3-4 times/ week), (4) usually ( $\geq 5$ times/week) and (5) unknown. The participants who selected 'unknown' (three participants) or gave no answer (three participants) were excluded from our analyses. Unless the participant chose 'no' or 'unknown', he or she was asked 'Which kind of spicy food do you like?', with four response categories: (1) less hot, (2) moderately hot, (3) very hot and (4) unknown.

\section{Determination of serum lipid profiles}

At the end of nutritional measurements, blood samples were collected via venepuncture on the next morning after at least $12 \mathrm{~h}$ of fasting ${ }^{(33,34)}$; samples were frozen and stored at $-86^{\circ} \mathrm{C}$ for later laboratory analysis. A national laboratory in Beijing (medical laboratory accreditation certificate ISO 15189:2007) analysed all samples using strict quality control $^{(34-36)}$. Laboratory analysis methods for the serum lipid profiles including TC, TAG, LDL-cholesterol and HDL-cholesterol are described in detail elsewhere $^{(35)}$, and all lipid measurements were detected using the Hitachi 7600 automated analyzer (Hitachi Inc.).

\section{Measurements of physical activity and other covariates}

Physical activity measurement was performed using staffadministered questionnaires including the four categories of domestic, occupational, transportation and leisure activity, which have been evaluated previously ${ }^{(37)}$. Domestic activity was measured on the basis of the average hours per day spent in buying food, preparing food, washing clothes and sweeping rooms during the previous week; occupational activity was categorised into three levels (light, moderate and heavy intensity) based on respondents' job descriptions, and participants were asked about the activity levels in their occupations and the working hours during an average work week; transportation activity was measured on the basis of four means of commuting to and from work or school: walking, bicycling, bus or subway and motorised vehicle, and all transportation activities were reported in average hours per week; measurement of leisure activity was based on five sports types: (1) martial arts, (2) gymnastics, dancing or acrobatics, (3) jogging or swimming, (4) playing soccer, basketball or tennis and (5) playing badminton, volleyball or table tennis, and all leisure activities were reported in average hours per weekday and weekend day. Metabolic equivalent (MET)-h/week was estimated by multiplying the time spent in each activity by its specific MET value based on the Compendium of Physical Activities $^{(38)}$. A detailed description of the MET values for assessing physical activity has been presented elsewhere ${ }^{(39)}$.

Standing height was measured to the nearest $0 \cdot 1 \mathrm{~cm}$ using a portable SECA stadiometer, with the subject not wearing shoes, and weight was measured to the nearest $0 \cdot 1 \mathrm{~kg}$ using a calibrated beam balance, with the subject not wearing heavy clothes and shoes ${ }^{(35)}$. Other demographic and lifestyle covariates, including date of birth, sex, ethnicity, educational level, smoking status and alcohol consumption were collected by trained and experienced interviewers using a structured questionnaire.

\section{Statistical analysis}

Participants' characteristics were presented as the mean values with standard deviations or as the median values with interquartile ranges for continuous variables with or without normal distribution, and as percentages for categorical variables. Intakes of dietary components were expressed in $\mathrm{g} / \mathrm{d}$ as well as percentage of energy. ANOVA, non-parametric Kruskal-Wallis tests (continuous variables) and $\chi^{2}$ tests (categorical variables) were used to compare participants' characteristics according to the frequency of spicy food consumption. To examine the associations between the frequency of spicy food intake and serum lipid profiles, we used the statistical software MLwiN 2.36 to construct multilevel (three levels) mixed-effects linear regression models (serum lipid levels) with individuals (level 1) nested within community (level 2) and province (level 3). Further, the associations between serum lipid profiles and different kinds (degree of pungency) as well as the average amount of spicy food intake, were examined using the aforementioned multilevel models. The main analysis was replicated in four multilevel analyses: original model without any adjustments, model 2 adjusted for age, sex, nationality, education, smoking status, alcohol consumption, BMI and physical activity, model 3 additionally adjusted for dietary intake of total energy and vegetable intake, and model 4 additionally adjusted for dietary intake of carbohydrate, fat and cholesterol. Descriptive and difference analyses were carried out using SPSS version 20.0 (SPSS Inc.). All statistical tests were two-sided, and $P<0.05$ was considered statistically significant.

\section{Results}

Table 1 displays the participants' characteristics according to the frequency of spicy food consumption. Frequency of spicy food intake was significantly positively associated with the amount of spicy food intake $\left(P_{\text {for trend }}<0 \cdot 001\right)$. Compared with participants who consumed spicy food less frequently (1-2 times/week or less), those who consumed spicy food more frequently (3-4 times/week or more) were more likely to be younger, male, belong to an ethnic minority, smoke tobacco and consume 
Table 1. Characteristics of the study participants from the China Health and Nutrition Survey according to frequency of spicy food consumption (Mean values and standard deviations; medians and interquartile ranges (IQR))

\begin{tabular}{|c|c|c|c|c|c|c|}
\hline & \multicolumn{4}{|c|}{ Frequency of spicy food consumption } & \multirow[b]{2}{*}{ Total } & \multirow[b]{3}{*}{$P^{*}$} \\
\hline & $\begin{array}{c}\text { No } \\
\text { (0 times/week) }\end{array}$ & $\begin{array}{c}\text { Sometimes } \\
\text { (1-2 times/week) }\end{array}$ & $\begin{array}{c}\text { Often } \\
\text { (3-4 times/week) }\end{array}$ & $\begin{array}{c}\text { Usually } \\
\text { ( } \geq 5 \text { times/week) }\end{array}$ & & \\
\hline & Mean & Mean & Mean & Mean & Mean & \\
\hline No. participants & 1837 & 2723 & 1375 & 839 & 6774 & \\
\hline \multicolumn{7}{|l|}{ Spicy food intake (g/d) } \\
\hline Median & \multirow{2}{*}{$\begin{array}{c}0.0 \\
0.0-0.0\end{array}$} & \multirow{2}{*}{$\begin{array}{c}0.0 \\
0.0-29.7\end{array}$} & \multirow{2}{*}{$\begin{array}{c}16 \cdot 7 \\
0.0-46 \cdot 7\end{array}$} & \multirow{2}{*}{$\begin{array}{c}23 \cdot 3 \\
0.0-50 \cdot 0\end{array}$} & \multirow{2}{*}{$\begin{array}{c}0.0 \\
0.0-33.3\end{array}$} & $<0.001$ \\
\hline IQR & & & & & & \\
\hline Age (years) & $47.5 \quad 11.7$ & $46.4 \quad 11.7$ & $44.8 \quad 11.6$ & $45.0 \quad 11.3$ & $46 \cdot 1 \quad 11.7$ & $<0.001$ \\
\hline BMI $\left(\mathrm{kg} / \mathrm{m}^{2}\right)$ & 23.4 & 23.5 & $23 \cdot 3$ & 23.4 & 23.4 & 0.141 \\
\hline Female (\%) & 58.3 & $52 \cdot 1$ & $49 \cdot 0$ & 50.8 & 53.0 & $<0.001$ \\
\hline Han nationality (\%) & $92 \cdot 7$ & $93 \cdot 2$ & $81 \cdot 6$ & 77.9 & $88 \cdot 8$ & $<0.001$ \\
\hline Education (years) & 8.0 & $8 \cdot 2$ & $8 \cdot 5$ & $8 \cdot 3$ & $8 \cdot 2$ & 0.005 \\
\hline Current smoker (\%) & 24.5 & 31.7 & 35.5 & $35 \cdot 8$ & 31.0 & $<0.001$ \\
\hline Alcohol consumption (\%) & $24 \cdot 1$ & $37 \cdot 2$ & $41 \cdot 7$ & $40 \cdot 8$ & $35 \cdot 0$ & $<0.001$ \\
\hline Physical activities (MET-h/ & & & & & & \\
\hline Median & $104 \cdot 0$ & 107.9 & 97.0 & $105 \cdot 4$ & $103 \cdot 9$ & 0.140 \\
\hline IQR & $43 \cdot 5-203 \cdot 5$ & $49 \cdot 4-196 \cdot 0$ & $44 \cdot 3-193 \cdot 7$ & $43 \cdot 7-192 \cdot 0$ & $46 \cdot 1-196 \cdot 1$ & \\
\hline Total energy intake (kcal/o & & & & & & \\
\hline Median & 2040.9 & $2149 \cdot 8$ & 2171.4 & $2115 \cdot 8$ & $2126 \cdot 4$ & $<0.001$ \\
\hline IQR & $1677 \cdot 9-2458 \cdot 6$ & $1759 \cdot 0-2603 \cdot 9$ & $1782 \cdot 9-2600 \cdot 2$ & $1687 \cdot 6-2547 \cdot 9$ & $1732 \cdot 9-2559 \cdot 8$ & \\
\hline Carbohydrate intake $(\mathrm{g} / \mathrm{d})$ & & & & & & \\
\hline Median & $282 \cdot 4$ & $293 \cdot 2$ & $293 \cdot 3$ & $295 \cdot 8$ & 290.6 & $<0.001$ \\
\hline IQR & $220 \cdot 3-355 \cdot 5$ & $234 \cdot 5-367 \cdot 1$ & $237 \cdot 2-358 \cdot 1$ & $233 \cdot 9-367 \cdot 3$ & $231 \cdot 4-361.5$ & \\
\hline Carbohydrate (E\%) & $56 \cdot 1 \quad 10 \cdot 7$ & $56.2 \quad 10.5$ & $55.5 \quad 10.8$ & $57.0 \quad 10.9$ & $56 \cdot 1 \quad 10 \cdot 7$ & 0.017 \\
\hline Fat intake $(g / d)$ & & & & & & \\
\hline Median & 68.0 & $71 \cdot 8$ & $73 \cdot 3$ & $69 \cdot 2$ & 70.7 & $<0.001$ \\
\hline IQR & $50 \cdot 3-90 \cdot 5$ & $51 \cdot 8-96 \cdot 6$ & $51 \cdot 8-98 \cdot 8$ & $47 \cdot 7-95 \cdot 4$ & $50 \cdot 9-95 \cdot 3$ & \\
\hline Fat $(E \%)$ & $31 \cdot 2 \quad 10.5$ & $31.2 \quad 10.5$ & $31.7 \quad 10.9$ & $30.5 \quad 10.7$ & $31 \cdot 2 \quad 10 \cdot 6$ & 0.072 \\
\hline Cholesterol intake $(\mathrm{g} / \mathrm{d})$ & & & & & & \\
\hline Median & $227 \cdot 4$ & $225 \cdot 3$ & $225 \cdot 7$ & $203 \cdot 4$ & 221.5 & $<0.001$ \\
\hline IQR & $116 \cdot 2-369 \cdot 1$ & $120 \cdot 2-370 \cdot 2$ & $129 \cdot 1-369 \cdot 9$ & $100 \cdot 1-345 \cdot 4$ & $118 \cdot 0-367 \cdot 6$ & \\
\hline Salt intake $(g / d)$ & $7.5 \quad 4.6$ & $7 \cdot 3 \quad 4.4$ & $\begin{array}{ll}7.4 & 4.7\end{array}$ & $7 \cdot 3 \quad 4.2$ & $7.4 \quad 4.5$ & 0.342 \\
\hline Vegetable intake (g/d) & & & & & & \\
\hline Median & $300 \cdot 0$ & $310 \cdot 0$ & $333 \cdot 3$ & $306 \cdot 7$ & $313 \cdot 3$ & $<0.001$ \\
\hline IQR & $201 \cdot 3-411 \cdot 2$ & $216 \cdot 7-416 \cdot 7$ & $230 \cdot 0-445 \cdot 0$ & $221 \cdot 7-404 \cdot 7$ & $216 \cdot 7-416 \cdot 7$ & \\
\hline
\end{tabular}

MET, metabolic equivalent; E\%, percentage of energy.

* Differences across all categories were obtained by using ANOVA, the Kruskal-Wallis test or $x^{2}$ test.

$\dagger$ To convert energy in $\mathrm{kcal} / \mathrm{d}$ to $\mathrm{kJ} / \mathrm{d}$, multiply by 4.184 .

alcohol, and more likely to have higher intakes of total energy and vegetables. However, there were no significant differences in BMI, educational level, physical activities and dietary intakes of carbohydrate, fat and cholesterol, as well as in proportions of energy intake from carbohydrate or fat and salt intake between participants who consumed spicy food less frequently and the others.

Frequency of spicy food consumption was significantly positively associated with TAG in a dose-response manner ( $P_{\text {for trend }}<0.05$ ) after adjustment for potential confounders and control for clustering of data at multiple levels (individual, community and province). In contrast, multilevel-adjusted analyses showed a significantly inverse association between frequency of spicy food consumption and LDL-cholesterol $\left(P_{\text {for trend }}<0.05\right)$. Compared with participants who did not eat spicy food, significantly lower LDL-cholesterol levels in those who ate spicy food sometime (by $0.059 \mathrm{mmol} / \mathrm{l} ; 95 \%$ CI $-0.116,-0.002$; $P=0.046$ ) or often (by $0.110 \mathrm{mmol} / 1 ; 95 \%$ CI $-0.179,-0.041$; $P=0.002)$ were observed. In addition, a positive association was found between frequency of spicy food consumption and HDLcholesterol in the present study $\left(P_{\text {for }}\right.$ trend $\left.<0 \cdot 01\right)$; significantly higher HDL-cholesterol (by $0 \cdot 110 \mathrm{mmol} / \mathrm{l} ; 95 \%$ CI 0.031, 0.189; $P=0.007)$ was found in participants who usually ate spicy food ( $\geq 5$ times/week) compared with those who did not eat. Furthermore, a higher frequency of spicy food consumption was associated with a progressively lower LDL-cholesterol: HDL-cholesterol ratio $\left(P_{\text {for trend }}<0 \cdot 001\right)$. Compared with participants who did not eat spicy food, those who consumed it sometimes, often or usually had a lower LDL-cholesterol:HDLcholesterol ratio by 0.066 (95\% CI $-0 \cdot 122,-0 \cdot 010 ; P=0 \cdot 021), 0 \cdot 113$ (95\% CI $-0.180,-0.046 ; P=0.001)$ or 0.133 (95\% CI -0.211 , $-0 \cdot 055 ; P=0.001$ ), respectively (Table 2 ).

We further performed multilevel analyses according to the average amount of spicy food intake (Table 3). Similar to the frequency of spicy food consumption, the average amount of spicy food was positively associated with TAG $\left(P_{\text {for trend }}<0 \cdot 01\right)$ and inversely associated with LDL-cholesterol $\left(P_{\text {for trend }}<0 \cdot 01\right)$ and LDL-cholesterol:HDL-cholesterol ratio $\left(P_{\text {for trend }}<0 \cdot 01\right)$. In addition, compared with participants who did not consume spicy food, those who consumed a low amount of spicy food $(0 \cdot 1-25 \cdot 0 \mathrm{~g} / \mathrm{d})$ had lower TC by $0 \cdot 110 \mathrm{mmol} / \mathrm{l}(95 \% \mathrm{CI}-0 \cdot 178$, $-0 \cdot 042 ; P=0 \cdot 001)$. 
Table 2. Multilevel-adjusted associations between categories of frequency of spicy food consumption and serum lipid profiles* ( $\beta$-Coefficients and $95 \%$ confidence intervals)

\begin{tabular}{|c|c|c|c|c|c|c|c|c|c|}
\hline & \multicolumn{8}{|c|}{ Frequency of spicy food consumption ( $n$ 6774) } & \multirow[b]{3}{*}{$P_{\text {for trend }}$} \\
\hline & \multicolumn{2}{|c|}{ No (0 times/week) } & \multicolumn{2}{|c|}{ Sometimes (1-2 times/week) } & \multicolumn{2}{|c|}{ Often (3-4 times/week) } & \multicolumn{2}{|c|}{ Usually ( $\geq 5$ times/week) } & \\
\hline & $\beta$ & $95 \% \mathrm{Cl}$ & $\beta$ & $95 \% \mathrm{Cl}$ & $\beta$ & $95 \% \mathrm{Cl}$ & $\beta$ & $95 \% \mathrm{Cl}$ & \\
\hline No. participants & \multicolumn{2}{|r|}{1837} & \multicolumn{2}{|r|}{2723} & \multicolumn{2}{|r|}{1375} & \multicolumn{2}{|r|}{839} & \\
\hline $\mathrm{TC}(\mathrm{mmol} / \mathrm{l})$ & 4.86 & 1.00 & 4.80 & 0.99 & 4.77 & 0.99 & 4.82 & 0.93 & 0.213 \\
\hline Model $1 \dagger$ & & Ref. & -0.056 & $-0.115,0.003$ & -0.092 & $-0.162,-0.022$ & -0.042 & $-0.124,0.040$ & 0.078 \\
\hline Model $2 \ddagger$ & & Ref. & -0.032 & $-0.089,0.025$ & -0.044 & $-0.112,0.024$ & -0.004 & $-0.083,0.075$ & 0.615 \\
\hline Model $3 \S$ & & Ref. & -0.030 & $-0.087,0.027$ & -0.038 & $-0.106,0.030$ & -0.004 & $-0.083,0.075$ & 0.674 \\
\hline Model 4\| & & Ref. & -0.027 & $-0.083,0.029$ & -0.041 & $-0.109,0.027$ & 0.006 & $-0.073,0.085$ & 0.762 \\
\hline TAG $(\mathrm{mmol} / \mathrm{l})$ & 1.55 & 1.23 & 1.61 & 1.25 & 1.67 & 1.31 & 1.68 & 1.28 & 0.005 \\
\hline Model $1 \dagger$ & & Ref. & 0.049 & $-0.010,0.108$ & 0.092 & $0.022,0.162$ & 0.106 & $0.024,0.188$ & 0.002 \\
\hline Model $2 \ddagger$ & & Ref. & 0.034 & $-0.023,0.091$ & 0.069 & $0.001,0.137$ & 0.091 & $0.012,0.170$ & 0.010 \\
\hline Model $3 \S$ & & Ref. & 0.036 & $-0.021,0.093$ & 0.072 & $0.004,0.140$ & 0.090 & $0.011,0.169$ & 0.010 \\
\hline Model $4 \|$ & & Ref. & 0.036 & $-0.021,0.093$ & 0.066 & $-0.002,0.134$ & 0.091 & $0.012,0.170$ & 0.013 \\
\hline LDL-cholesterol (mmol/l) & 3.03 & 0.99 & 2.94 & 0.92 & $2 \cdot 86$ & 0.91 & 2.90 & 0.97 & $<0.001$ \\
\hline Model $1 \dagger$ & & Ref. & -0.093 & $-0.152,-0.034$ & -0.180 & $-0.250,-0.110$ & -0.135 & $-0.216,-0.054$ & $<0.001$ \\
\hline Model $2 \ddagger$ & & Ref. & -0.063 & $-0.120,-0.006$ & -0.118 & $-0.187,-0.049$ & -0.078 & $-0.158,0.002$ & 0.005 \\
\hline Model $3 \S$ & & Ref. & -0.062 & $-0.119,-0.005$ & -0.111 & $-0.180,-0.042$ & -0.077 & $-0.157,0.003$ & 0.007 \\
\hline Model 4\| & & Ref. & -0.059 & $-0.116,-0.002$ & -0.110 & $-0.179,-0.041$ & -0.072 & $-0.152,0.008$ & 0.010 \\
\hline HDL-cholesterol (mmol/l) & 1.41 & 0.36 & 1.41 & 0.37 & 1.42 & 0.40 & 1.45 & 0.38 & 0.009 \\
\hline Model $1 \dagger$ & & Ref. & 0.006 & $-0.053,0.065$ & 0.029 & $-0.041,0.099$ & 0.104 & $0.022,0.186$ & 0.017 \\
\hline Model $2 \ddagger$ & & Ref. & 0.028 & $-0.029,0.085$ & 0.054 & $-0.014,0.122$ & 0.102 & $0.023,0.181$ & 0.009 \\
\hline Model $3 \S$ & & Ref. & 0.024 & $-0.033,0.081$ & 0.049 & $-0.019,0.117$ & 0.102 & $0.023,0.181$ & 0.010 \\
\hline Model 4\| & & Ref. & 0.027 & $-0.030,0.084$ & 0.051 & $-0.017,0.119$ & 0.110 & $0.031,0.189$ & 0.006 \\
\hline LDL-cholesterol:HDL-cholesterol ratio & $2 \cdot 26$ & 0.86 & $2 \cdot 19$ & 0.81 & $2 \cdot 13$ & 0.82 & $2 \cdot 11$ & 0.83 & $<0.001$ \\
\hline Model $1 \dagger$ & & Ref. & -0.078 & $-0.137,-0.019$ & -0.149 & $-0.219,-0.079$ & -0.174 & $-0.255,-0.093$ & $<0.001$ \\
\hline Model $2 \ddagger$ & & Ref. & -0.072 & $-0.128,-0.016$ & -0.123 & $-0.190,-0.056$ & -0.136 & $-0.214,-0.058$ & $<0.001$ \\
\hline Model $3 \S$ & & Ref. & -0.068 & $-0.124,-0.012$ & -0.114 & $-0.181,-0.047$ & -0.135 & $-0.213,-0.057$ & $<0.001$ \\
\hline Model 4\| & & Ref. & -0.066 & $-0.122,-0.010$ & -0.113 & $-0.180,-0.046$ & -0.133 & $-0.211,-0.055$ & $<0.001$ \\
\hline
\end{tabular}

TC, total cholesterol; Ref., referent values.

* All of the models were constructed by using multilevel (three levels) mixed-effects linear regression with iterative generalised least-squares method.

† Model 1: original model without any adjustments.

$\ddagger$ Model 2: adjusted for age, sex (male or female), nationality (Han or others), education ( $\leq 6 \cdot 0,7 \cdot 0-9.0$ or $>9.0$ years), smoking status (current or not current), alcohol consumption (yes or no), BMI (<18.5, 18.5-24.9, 25-29.9, $\geq 30 \mathrm{~kg} / \mathrm{m}^{2}$ and missing) and physical activity (quartile).

$\S$ Model 3: adjusted as for model 2 plus dietary intake of total energy (quartile) and vegetables (quartile).

II Model 4: adjusted as for model 3 plus dietary intakes of carbohydrate (quartile), fat (quartile) and cholesterol (quartile).

Associations between different degrees of pungency in spicy food consumed and serum lipid profiles are shown in Table 4. No association between the degree of pungency in spicy food consumed and serum TC was observed in multileveladjusted analyses. Compared with participants who did not eat spicy food, those who ate moderately hot food exhibited significantly higher HDL-cholesterol level (by $0.091 \mathrm{mmol} / \mathrm{l}$; $95 \% \mathrm{CI} 0 \cdot 030,0 \cdot 152 ; P=0 \cdot 003$ ). In contrast, a positive association between the degree of pungency and serum TAG was found in the present study $(P<0.05)$. Participants who ate less hot (by $0.069 \mathrm{mmol} / 1 ; 95 \% \mathrm{CI}-0.128,-0.010 ; P=0.022$ ), moderately hot (by $0.072 \mathrm{mmol} / 1 ; 95 \% \mathrm{CI}-0.133,-0.011 ; P=0.021$ ) and very hot (by $0.135 \mathrm{mmol} / 1 ; 95 \% \mathrm{CI}-0.239,-0.031 ; P=0.011$ ) food presented significantly lower serum LDL-cholesterol than those who did not eat spicy food, which showed a statistically linear trend $\left(P_{\text {for trend }}<0.01\right)$. Further, the degree of pungency in spicy food was observed to be inversely associated with the LDL-cholesterol:HDL-cholesterol ratio in the current study $\left(P_{\text {for trend }}<0.01\right)$; LDL-cholesterol:HDL-cholesterol ratio in participants who consumed less hot (by 0.076; $95 \%$ CI -0.134 , $-0 \cdot 018 ; P=0.010$ ) and moderately hot (by $0 \cdot 117 ; 95 \% \mathrm{CI}-0 \cdot 177$, $-0.057 ; P<0.001)$ food were significantly lower than those who did not eat spicy food.

\section{Discussion}

To our knowledge, no published studies that examined the relationship between spicy food consumption and serum lipids have enrolled a geographically diverse and large-scale participant population. In our study, we examined whether spicy food consumption and the degree of pungency in spicy food are associated with serum lipid profiles in a Chinese population. We found that the frequency of spicy food consumption and the average amount of spicy food intake were inversely associated with LDL-cholesterol. In addition, it was observed that the frequency of spicy food consumption and the average amount of spicy food intake were inversely associated with LDL-cholesterol: HDL-cholesterol ratio, which suggests that spicy food intake may be an alternative choice to reduce the risk for CVD. Further analysis of the results showed that those associations might be due to bioactive components responsible for pungency in spicy food. In contrast, our results showed that the frequency of spicy food consumption and the average amount of spicy food intake were positively associated with serum TAG.

One study ${ }^{(40)}$ collected information on the pattern of spice use, as well as the frequency and quantity of consumption of seventeen spices routinely used in Indian cuisine, and showed 
Table 3. Multilevel-adjusted associations between categories of average amount of spicy food intake and serum lipid profiles* ( $\beta$-Coefficients and $95 \%$ confidence intervals)

\begin{tabular}{|c|c|c|c|c|c|c|c|c|c|}
\hline & \multicolumn{8}{|c|}{ Average amount of spicy food intake ( $n 6774)$} & \multirow[b]{3}{*}{$P_{\text {for trend }}$} \\
\hline & \multicolumn{2}{|c|}{$0.0 \mathrm{~g} / \mathrm{d}$} & \multicolumn{2}{|c|}{$0.1-25.0 \mathrm{~g} / \mathrm{d}$} & \multicolumn{2}{|c|}{$25.1-50.0 \mathrm{~g} / \mathrm{d}$} & \multicolumn{2}{|c|}{$>50.0 \mathrm{~g} / \mathrm{d}$} & \\
\hline & $\beta$ & $95 \% \mathrm{Cl}$ & $\beta$ & $95 \% \mathrm{Cl}$ & $\beta$ & $95 \% \mathrm{Cl}$ & $\beta$ & $95 \% \mathrm{Cl}$ & \\
\hline No. participants & \multicolumn{2}{|r|}{3846} & \multicolumn{2}{|r|}{951} & \multicolumn{2}{|r|}{1105} & \multicolumn{2}{|r|}{872} & \\
\hline $\mathrm{TC}(\mathrm{mmol} / \mathrm{l})$ & 4.84 & 1.00 & $4 \cdot 71$ & 0.93 & 4.82 & 1.00 & 4.77 & 0.98 & 0.384 \\
\hline Model $1 \dagger$ & & Ref. & -0.131 & $-0.202,-0.060$ & -0.024 & $-0.091,0.043$ & -0.071 & $-0.144,0.002$ & 0.046 \\
\hline Model $2 \ddagger$ & & Ref. & -0.096 & $-0.164,-0.028$ & 0.004 & $-0.060,0.068$ & -0.061 & $-0.131,0.009$ & 0.161 \\
\hline Model $3 \S$ & & Ref. & -0.105 & $-0.173,-0.037$ & 0.008 & $-0.056,0.072$ & -0.041 & $-0.112,0.030$ & 0.326 \\
\hline Model $4 \|$ & & Ref. & $-0 \cdot 110$ & $-0.178,-0.042$ & -0.003 & $-0.067,0.061$ & -0.052 & $-0.123,0.019$ & 0.172 \\
\hline TAG $(\mathrm{mmol} / \mathrm{l})$ & 1.59 & 1.23 & 1.56 & 1.19 & 1.70 & 1.34 & 1.69 & 1.36 & 0.003 \\
\hline Model $1 \dagger$ & & Ref. & -0.022 & $-0.093,0.049$ & 0.088 & $0.021,0.155$ & 0.084 & $0.011,0.157$ & 0.004 \\
\hline Model $2 \ddagger$ & & Ref. & 0.003 & $-0.065,0.071$ & 0.092 & $0.028,0.156$ & 0.080 & $0.010,0.150$ & 0.002 \\
\hline Model $3 \S$ & & Ref. & 0.002 & $-0.066,0.070$ & 0.089 & $0.025,0.153$ & 0.089 & $0.017,0.161$ & 0.002 \\
\hline Model $4 \|$ & & Ref. & -0.004 & $-0.072,0.064$ & 0.081 & $0.017,0.145$ & 0.086 & $0.014,0.158$ & 0.003 \\
\hline LDL-cholesterol (mmol/l) & 2.99 & 0.96 & $2 \cdot 86$ & 0.94 & 2.94 & 0.93 & $2 \cdot 86$ & 0.88 & 0.010 \\
\hline Model $1 \dagger$ & & Ref. & $-0 \cdot 138$ & $-0.209,-0.067$ & -0.045 & $-0.112,0.022$ & -0.134 & $-0.207,-0.061$ & $<0.001$ \\
\hline Model $2 \ddagger$ & & Ref. & $-0 \cdot 100$ & $-0.169,-0.031$ & -0.017 & $-0.081,0.047$ & -0.119 & $-0.190,-0.048$ & 0.003 \\
\hline Model $3 \S$ & & Ref. & -0.109 & $-0.178,-0.040$ & -0.011 & $-0.076,0.054$ & $-0 \cdot 100$ & $-0.172,-0.028$ & 0.014 \\
\hline Model 4\| & & Ref. & $-0 \cdot 109$ & $-0.178,-0.040$ & -0.016 & $-0.081,0.049$ & -0.106 & $-0.178,-0.034$ & 0.008 \\
\hline HDL-cholesterol (mmol/l) & 1.42 & 0.37 & 1.42 & 0.36 & 1.40 & 0.37 & 1.44 & 0.38 & 0.268 \\
\hline Model $1 \dagger$ & & Ref. & 0.009 & $-0.062,0.080$ & -0.048 & $-0.115,0.019$ & 0.064 & $-0.009,0.137$ & 0.517 \\
\hline Model $2 \ddagger$ & & Ref. & -0.017 & $-0.085,0.051$ & -0.041 & $-0.105,0.023$ & 0.061 & $-0.009,0.131$ & 0.491 \\
\hline Model $3 \S$ & & Ref. & -0.017 & $-0.086,0.052$ & -0.037 & $-0.101,0.027$ & 0.054 & $-0.018,0.126$ & 0.587 \\
\hline Model 4\| & & Ref. & -0.018 & $-0.087,0.051$ & -0.039 & $-0.104,0.026$ & 0.048 & $-0.024,0.120$ & 0.677 \\
\hline LDL-cholesterol:HDL-cholesterol ratio & $2 \cdot 22$ & 0.84 & $2 \cdot 12$ & 0.84 & $2 \cdot 21$ & 0.81 & 2.09 & 0.76 & 0.003 \\
\hline Model $1 \dagger$ & & Ref. & $-0 \cdot 117$ & $-0.188,-0.046$ & -0.010 & $-0.077,0.057$ & -0.157 & $-0.230,-0.084$ & $<0.001$ \\
\hline Model $2 \ddagger$ & & Ref. & -0.072 & $-0.139,-0.005$ & 0.007 & $-0.056,0.070$ & -0.146 & $-0.215,-0.077$ & 0.001 \\
\hline Model $3 \S$ & & Ref. & -0.081 & $-0.149,-0.013$ & 0.010 & $-0.053,0.073$ & -0.122 & $-0.193,-0.051$ & 0.009 \\
\hline Model 4\| & & Ref. & -0.082 & $-0.150,-0.014$ & 0.006 & $-0.058,0.070$ & -0.126 & $-0.197,-0.055$ & 0.006 \\
\hline
\end{tabular}

TC, total cholesterol; Ref, referent values.

* All of the models were constructed using multilevel (three levels) mixed-effects linear regression with the iterative generalised least-squares method.

† Model 1: original model without any adjustments.

$\ddagger$ Model 2: adjusted for age, sex (male or female), nationality (Han or others), education ( $\leq 6 \cdot 0,7 \cdot 0-9.0$ or $>9.0$ years), smoking status (current or not current), alcohol consumption (yes or no), BMI $\left(<18.5,18.5-24.9,25-29.9, \geq 30 \mathrm{~kg} / \mathrm{m}^{2}\right.$ and missing) and physical activity (quartile).

$\S$ Model 3: adjusted as for model 2 plus dietary intake of total energy (quartile) and vegetables (quartile).

II Model 4: adjusted as for model 3 plus dietary intakes of carbohydrate (quartile), fat (quartile) and cholesterol (quartile).

that the frequency of consumption of spice-containing dishes facilitated in quantifying spice intake at the individual level. A previous study $^{(15)}$ using a similar questionnaire on frequency of spicy food consumption carried out a validated pilot investigation on dietary habits from repeated collections of dietary information, and found that intakes of dietary factors, including spicy food, were highly consistent over time ( 1.4 years). Moreover, the frequency of spicy food consumption was found to be positively associated with the average amount of spicy food intake according to dietary-intake recall $\left(P_{\text {for trend }}<0 \cdot 001\right)$ in our study, which indicates that the assessment of frequency of spicy food consumption is consistent, reliable and informative, at least for ranking participant behaviours and estimating relative risks.

The effects of chillies on the modulation of lipid metabolism have been extensively investigated in high-fat animal models. Kim \& Park $^{(16)}$ have reported that serum TAG, TC and LDLcholesterol in mice on a $45 \%$ high-fat diet supplemented with green-pepper juice $(10 \mathrm{ml} / \mathrm{kg}$ per d) were significantly lower than those not fed green-pepper juice. Kwon et al. ${ }^{(41)}$ have shown that feeding a $1 \%$ red-pepper powder $+1 \%$ cholesterol diet to rabbits led to a significant decrease in TC, TAG, LDLcholesterol and VLDL-cholesterol, and a significant increase in HDL-cholesterol in comparison with the control. The present human study showed that serum LDL-cholesterol and LDL-cholesterol:HDL-cholesterol ratio were inversely associated with spicy food consumption; also, a significant decrease in TC and a significant increase in HDL-cholesterol were found in subgroups of spicy food consumption in comparison with those without spicy food intake. Considering the fact that modulation of lipid metabolism plays an important role in the process of CVD development $^{(1,6,35,36)}$, chilli consumption may be an alternative choice for cardiovascular health protection. Although improvement of lipid metabolism was not confirmed in other human studies, positive findings were reported from the regular consumption of chillies for 4 weeks on the resistance of serum lipoproteins to oxidation in healthy adults ${ }^{(24)}$, as well as on the increase of serum apoB with chilli supplementation in women having $\mathrm{GDM}^{(23)}$. However, another clinical study with a chilli diet has observed no obvious beneficial effects on lipid metabolism during 3 weeks of intervention ${ }^{(25)}$. The major differences between the present study and those clinical studies were research type, study population and sample size. These studies ${ }^{(24,25)}$ aimed to find changes in serum lipids and lipoproteins after only 3 or 4 weeks of chilli-food intervention, which would prove more difficult in terms of obtaining 
Table 4. Multilevel-adjusted associations between categories of degree of pungency in spicy food and serum lipid profiles* ( $\beta$-Coefficients and $95 \%$ confidence intervals)

\begin{tabular}{|c|c|c|c|c|c|c|c|c|c|}
\hline & \multicolumn{8}{|c|}{ Degree of pungency in spicy food ( $n$ 6761) } & \multirow[b]{3}{*}{$P_{\text {for trend }}$} \\
\hline & \multicolumn{2}{|c|}{ Non-eating } & \multicolumn{2}{|c|}{ Low pungency } & \multicolumn{2}{|c|}{ Moderate pungency } & \multicolumn{2}{|c|}{ High pungency } & \\
\hline & $\beta$ & $95 \% \mathrm{Cl}$ & $\beta$ & $95 \% \mathrm{Cl}$ & $\beta$ & $95 \% \mathrm{Cl}$ & $\beta$ & $95 \% \mathrm{Cl}$ & \\
\hline No. participants & \multicolumn{2}{|r|}{1837} & \multicolumn{2}{|r|}{2375} & \multicolumn{2}{|r|}{2135} & \multicolumn{2}{|r|}{414} & \\
\hline TC $(\mathrm{mmol} / \mathrm{l})$ & 4.86 & 1.00 & 4.81 & 0.99 & 4.79 & 0.97 & 4.70 & 0.96 & 0.003 \\
\hline Model $1 \dagger$ & & Ref. & -0.048 & $-0.109,0.013$ & -0.064 & $-0.126,-0.002$ & $-0 \cdot 161$ & $-0.267,-0.055$ & 0.003 \\
\hline Model $2 \ddagger$ & & Ref. & -0.039 & $-0.097,0.019$ & -0.008 & $-0.068,0.052$ & $-0 \cdot 109$ & $-0.211,-0.007$ & 0.257 \\
\hline Model $3 \S$ & & Ref. & -0.037 & $-0.095,0.021$ & -0.004 & $-0.064,0.056$ & $-0 \cdot 106$ & $-0.208,-0.004$ & 0.304 \\
\hline Model 4\| & & Ref. & -0.034 & $-0.092,0.024$ & -0.003 & $-0.063,0.057$ & -0.099 & $-0.201,0.003$ & 0.351 \\
\hline TAG $(\mathrm{mmol} / \mathrm{l})$ & 1.55 & 1.23 & 1.63 & 1.29 & 1.64 & 1.24 & 1.70 & 1.36 & 0.026 \\
\hline Model 1† & & Ref. & 0.061 & $0.000,0.122$ & 0.073 & $0.011,0.135$ & 0.119 & $0.012,0.226$ & 0.008 \\
\hline Model $2 \ddagger$ & & Ref. & 0.040 & $-0.019,0.099$ & 0.061 & $0.000,0.122$ & 0.094 & $-0.009,0.197$ & 0.023 \\
\hline Model 3§ & & Ref. & 0.042 & $-0.017,0.101$ & 0.064 & $0.003,0.125$ & 0.093 & $-0.010,0.196$ & 0.021 \\
\hline Model 4\| & & Ref. & 0.040 & $-0.019,0.099$ & 0.061 & $0.000,0.122$ & 0.096 & $-0.007,0.199$ & 0.022 \\
\hline LDL-cholesterol (mmol/l) & 3.03 & 0.99 & 2.94 & 0.92 & $2 \cdot 89$ & 0.93 & $2 \cdot 83$ & 0.91 & $<0.001$ \\
\hline Model 1† & & Ref. & -0.092 & $-0.153,-0.031$ & $-0 \cdot 145$ & $-0.207,-0.083$ & -0.218 & $-0.324,-0.112$ & $<0.001$ \\
\hline Model $2 \ddagger$ & & Ref. & -0.074 & $-0.133,-0.015$ & -0.078 & $-0.139,-0.017$ & $-0 \cdot 144$ & $-0.248,-0.040$ & 0.003 \\
\hline Model $3 \S$ & & Ref. & -0.072 & $-0.131,-0.013$ & -0.075 & $-0.136,-0.014$ & $-0 \cdot 140$ & $-0.244,-0.036$ & 0.004 \\
\hline Model $4 \|$ & & Ref. & -0.069 & $-0.128,-0.010$ & -0.072 & $-0.133,-0.011$ & -0.135 & $-0.239,-0.031$ & 0.005 \\
\hline HDL-cholesterol (mmol/l) & 1.41 & 0.36 & 1.41 & 0.37 & 1.44 & 0.38 & 1.37 & 0.40 & 0.144 \\
\hline Model $1 \dagger$ & & Ref. & 0.011 & $-0.050,0.072$ & 0.073 & $0.011,0.135$ & -0.101 & $-0.208,0.006$ & 0.512 \\
\hline Model $2 \ddagger$ & & Ref. & 0.026 & $-0.033,0.085$ & 0.094 & $0.033,0.155$ & -0.086 & $-0.190,0.018$ & 0.207 \\
\hline Model $3 \S$ & & Ref. & 0.023 & $-0.036,0.082$ & 0.089 & $0.028,0.150$ & -0.085 & $-0.188,0.018$ & 0.245 \\
\hline Model 4\| & & Ref. & 0.027 & $-0.032,0.086$ & 0.091 & $0.030,0.152$ & -0.083 & $-0.186,0.020$ & 0.225 \\
\hline LDL-cholesterol:HDL-cholesterol ratio & $2 \cdot 26$ & 0.86 & $2 \cdot 19$ & 0.80 & $2 \cdot 12$ & 0.82 & $2 \cdot 19$ & 0.86 & 0.071 \\
\hline Model 1† & & Ref. & -0.082 & $-0.143,-0.021$ & -0.158 & $-0.220,-0.096$ & -0.074 & $-0.180,0.032$ & $<0.001$ \\
\hline Model $2 \ddagger$ & & Ref. & -0.080 & $-0.138,-0.022$ & $-0 \cdot 126$ & $-0.186,-0.066$ & -0.037 & $-0.139,0.065$ & 0.002 \\
\hline Model $3 \S$ & & Ref. & -0.076 & $-0.134,-0.018$ & $-0 \cdot 119$ & $-0.179,-0.059$ & -0.034 & $-0.136,0.068$ & 0.004 \\
\hline Model 4\| & & Ref. & -0.076 & $-0.134,-0.018$ & -0.117 & $-0.177,-0.057$ & -0.030 & $-0.132,0.072$ & 0.005 \\
\hline
\end{tabular}

TC, total cholesterol; Ref, referent values.

* All of the models were constructed using multilevel (three levels) mixed-effects linear regression with iterative generalised least-squares method.

$\dagger$ Model 1: original model without any adjustments.

$\ddagger$ Model 2: adjusted for age, sex (male or female), nationality (Han or others), education ( $\leq 6.0,7 \cdot 0-9.0$ or $>9.0$ years), smoking status (current or not current), alcohol consumption (yes or no), BMI $\left(<18.5,18.5-24.9,25-29.9, \geq 30 \mathrm{~kg} / \mathrm{m}^{2}\right.$ and missing) and physical activity (quartile).

$\S$ Model 3: adjusted as for model 2 plus dietary intake of total energy (quartile) and vegetables (quartile).

II Model 4: adjusted as for model 3 plus dietary intakes of carbohydrate (quartile), fat (quartile) and cholesterol (quartile).

significant results than checking the association between spicy food consumption and serum lipids. The strengths of our study are the large sample size and the cautious analyses from different dimensions (frequency and amount of spicy food intake, and the degree of pungency of spicy food consumed). Considering the inherent defects of a cross-sectional study, further investigations are warranted to confirm the relation between spicy food consumption and lipid metabolism. It is noteworthy that the use of spice has been suggested in Western countries in order to reduce salt consumption that could increase cardiovascular risk. However, a significant association between spicy food and salt consumption was not found in the present study.

The hypocholesterolaemic effect of spicy food consumption observed in the present study may be due to the capsaicinoids or a combined effect with other phytochemicals such as ascorbic acid, tocopherol, carotenoids and phenolic compounds present in the chilli. Capsaicinoids, a group of pungent compounds found in chillies, contain capsaicin, dihydrocapsaicin, nordihydrocapsaicin, homodihydrocapsaicin and homocapsaicin, etc. ${ }^{(20)}$. Capsaicinoids have been found to increase energy expenditure ${ }^{(42)}$, reduce adipose tissue ${ }^{(43,44)}$, decrease oxidative stress ${ }^{(45)}$ and enhance lipid metabolism ${ }^{(19,46,47)}$. The possible mechanism of the hypocholesterolaemic effect could be through decreased cholesterol absorption accompanied by increased excretion of cholesterol and bile acids in the faeces by regulating the expression of hepatic LDL receptors ${ }^{(17,45)}$. Accumulated evidence demonstrates that dietary capsaicinoids can effectively up-regulate the expression of hepatic cholester- $7 \alpha$-hydroxylase and stimulate the conversion of cholesterol to bile acids ${ }^{(17,20,22,48)}$, whereas they do not affect the expression of 3-hydroxy-3-methylglutaryl-CoA reductase that is involved in cholesterol synthesis ${ }^{(20)}$. Our results are consistent with the above-mentioned results from animal studies and showed that the degree of pungency in spicy food was inversely associated with LDL-cholesterol and LDL-cholesterol:HDL-cholesterol ratio, indicating that capsaicinoids might play a central role in the hypocholesterolaemic effect. In addition, capsanthin, the major carotenoid present in chillies, attenuates obesity-induced inflammation $^{(49)}$, inhibits LDL oxidation ${ }^{(50)}$ and raises plasma HDL-cholesterol levels ${ }^{(51)}$. Moreover, chilli peppers are a remarkable source of phenolic compounds particularly flavonoids, quercetin and luteolin ${ }^{(52)}$, and these compounds exhibit antioxidant, anti-inflammatory and hypolipidaemic activities, which are closely linked to the prevention and management of $\mathrm{CVD}^{(53)}$.

Chilli peppers and their extracts, particularly capsaicinoids, showed TAG-lowering properties in animal studies $(16,17,20,54)$. However, positive associations were found between serum TAG concentration and the frequency of spicy food consumption as well as the average amount of spicy food intake in the present study. 
The potential explanation may be that increased total energy and fat intake in humans was associated with spicy food intake. The type of dyslipidaemia (mainly hypertriacylglycerolaemia and low HDL-cholesterol) in Chinese adults is different from those in Western countries (mainly hypercholesteraemia and high LDL-cholesterol) ${ }^{(3,4)}$, probably because of low dietary fat and cholesterol intake and high carbohydrate intake in Chinese adults $^{(4)}$. In contrast with meat being part of the staple food in a typical Western diet, plain and tasteless rice- or flour-based foods are staples for the Chinese population ${ }^{(55)}$. Chillies can transform those insipid foods into attractive, appetising meals by imparting a pleasing flavour, colour and pungency, which may result in increasing total energy and carbohydrate intakes in Chinese people $^{(15,55)}$ (our data partly support this theory). In addition, apart from fresh chillies, some chilli products are popular in China, including not only chilli powder but also mixtures known as chilli sauce, chilli oil and pickles with substantial salt and/or TAG content $^{(15)}$. Further, given the fact that spicy foods are more meatbased rather than vegetable-based in Chinese cuisines ${ }^{(56)}$, such as hot pot and beef jerky, more salt and/or oil are consumed for the improvement of flavour and preservation. Actually, Sun et al. ${ }^{(55)}$ have showed that spicy food intake is a potential risk factor for obesity in the Chinese population, especially for central obesity in males, which partly agreed with the findings from a Korean study by Yoo et $a l .{ }^{(57)}$. Increasing the energy and fat intake would contribute to elevated TAG levels in blood and the accumulation of fat in tissue. However, not all hypolipidaemic mechanisms of chilli peppers and their extracts are completely known and understood, and more research is needed to elucidate these functional mechanisms, especially of TAG metabolism.

Some limitations of the present study should be mentioned. First, the cholesterol-reducing activity of chillies and related mechanisms were reported in animal studies, which are consistent with our results, but the serum TAG -lowering effect of spicy food in animal studies was contradictory to our results. It is difficult to accurately assess oil intake by individual dietary recall even when using inventory change combined with the weighting method, because the amount of oil and fat in processed spicy food and of those left in the residue of food was uncertain. Future studies might develop more effective and accurate methods to estimate oil intake for exploration of the association between spicy food and serum TAG after controlling for potential confounders from dietary oil consumption. Second, though this longitudinal survey has been conducted in different years, information about the frequency of spicy food consumption was only collected in the 2009 survey. On the basis of the cross-sectional analysis, it is useful to outline the associations between spicy food consumption and serum lipid profiles, but it cannot establish their causal relationship. Otherwise, the pathophysiological mechanisms underlying the effect of spicy food consumption on serum lipid profiles cannot be investigated in the present observational study.

\section{Conclusions}

To our knowledge, this cross-sectional study is the first one carried out in a geographically diverse and large-scale population that shows frequency of spicy food consumption to be inversely related to LDL-cholesterol and LDL-cholesterol:HDL-cholesterol ratio, and positively to HDL-cholesterol. These associations are reinforced further by the average degree of pungency in spicy food and the amount of spicy food intake, which indicate that spicy food consumption may be a protective factor for cholesterol metabolism in the Chinese adult population and that bioactive components responsible for pungency in spicy food may play an important role in these interactions. However, positive associations between spicy food consumption and serum TAG concentration are found in the present study, which may be due to increasing energy and oil intake accompanying spicy food consumption. The findings from our study suggest that spicy food consumption may contribute to cardiovascular health, and additional research is needed to confirm this finding as well as the potential roles of spicy food in cholesterol and TAG metabolisms.

\section{Acknowledgements}

This research used data from the 2009 phase of the China Health and Nutrition Survey (CHNS). The authors are thankful to all subjects who participated in the study. The authors also thank the team at the National Institute for Nutrition and Health, Chinese Center for Disease Control and Prevention and the Carolina Population Center, University of North Carolina at Chapel Hill, and the China-Japan Friendship Hospital, Ministry of Health, for supporting CHNS 2009.

This research received no specific grant from any funding agency in the public, commercial, or non-profit sectors.

The authors' contributions were as follows: B. Z., Y. Z., Y. X. and T. H. contributed to the conception and design of the research; Y. X., T. H. and A. Z. analysed the data and interpreted results; Y. X. acquired the data from the open database for CHNS (http://www. cpc.unc.edu/projects/china/data/datasets/longitudinal/datasets); Y. X., T. H., W. Z. and K. Y. drafted the manuscript; all authors critically revised the paper and approved the final version of the manuscript.

The authors declare that there are no conflicts of interest.

\section{References}

1. Laslett LJ, Alagona P Jr, Clark BA 3rd, et al. (2012) The worldwide environment of cardiovascular disease: prevalence, diagnosis, therapy, and policy issues: a report from the American College of Cardiology. J Am Coll Cardiol 60, Suppl. 25, S1-S49.

2. Stone NJ, Robinson JG, Lichtenstein AH, et al. (2014) 2013 ACC/AHA guideline on the treatment of blood cholesterol to reduce atherosclerotic cardiovascular risk in adults: a report of the American College of Cardiology/American Heart Association Task Force on Practice Guidelines. Circulation 129, Suppl. 2, S1-S45.

3. Shao X, Yang W, Shao X, et al. (2016) The role of active brown adipose tissue (aBAT) in lipid metabolism in healthy Chinese adults. Lipids Health Dis 15, 138.

4. Shao X, Yang W, Shao X, et al. (2016) The prevalence, awareness, treatment and control of dyslipidemia among adults in China. Atherosclerosis 248, 2-9.

5. Yang W, Xiao J, Yang Z, et al. (2012) Serum lipids and lipoproteins in Chinese men and women. Circulation $\mathbf{1 2 5}$, $2212-2221$. 
6. Qi L, Ding X, Tang W, et al. (2015) Prevalence and risk factors associated with dyslipidemia in Chongqing, China. Int $J$ Environ Res Public Health 12, 13455-13465.

7. Claas SA \& Arnett DK (2016) The role of healthy lifestyle in the primordial prevention of cardiovascular disease. Curr Cardiol Rep 18, 56.

8. Kowalska K \& Olejnik A (2016) Current evidence on the health-beneficial effects of berry fruits in the prevention and treatment of metabolic syndrome. Curr Opin Clin Nutr Metab Care 19, 446-452.

9. Huang H, Xie Z, Boue SM, et al. (2013) Cholesterol-lowering activity of soy-derived glyceollins in the golden Syrian hamster model. J Agric Food Chem 61, 5772-5782.

10. Bahadoran Z, Golzarand M, Mirmiran P, et al. (2013) The association of dietary phytochemical index and cardiometabolic risk factors in adults: Tehran Lipid and Glucose Study. J Hum Nutr Diet 26, Suppl. 1, 145-153.

11. Szolcsanyi J (2004) Forty years in capsaicin research for sensory pharmacology and physiology. Neuropeptides 38, 377-384.

12. Kim JS, Lee WM, Rhee HC, et al. (2016) Red paprika (Capsicum annuum L.) and its main carotenoids, capsanthin and beta-carotene, prevent hydrogen peroxide-induced inhibition of gap-junction intercellular communication. Chem Biol Interact 254, 146-155.

13. Ritota M, Marini F, Sequi $\mathrm{P}$, et al. (2010) Metabolomic characterization of Italian sweet pepper (Capsicum annum L.) by means of HRMAS-NMR spectroscopy and multivariate analysis. J Agric Food Chem 58, 9675-9684.

14. Li J, Wang R \& Xiao C (2014) Association between chilli food habits with iron status and insulin resistance in a Chinese population. J Med Food 17, 472-478.

15. Lv J, Qi L, Yu C, et al. (2015) Consumption of spicy foods and total and cause specific mortality: population based cohort study. BMJ 351, h3942.

16. Kim NH \& Park SH (2015) Evaluation of green pepper (Capsicum annuum L.) juice on the weight gain and changes in lipid profile in C57BL/6 mice fed a high-fat diet. J Sci Food Agric 95, 79-87.

17. Liang YT, Tian XY, Chen JN, et al. (2013) Capsaicinoids lower plasma cholesterol and improve endothelial function in hamsters. Eur J Nutr 52, 379-388.

18. Huang W, Cheang WS, Wang X, et al. (2014) Capsaicinoids but not their analogue capsinoids lower plasma cholesterol and possess beneficial vascular activity. J Agric Food Chem 62, 8415-8420.

19. Tani Y, Fujioka T, Sumioka M, et al. (2004) Effects of capsinoid on serum and liver lipids in hyperlipidemic rats. $J$ Nutr Sci Vitaminol (Tokyo) 50, 351-355.

20. Zhang L, Zhou M, Fang G, et al. (2013) Hypocholesterolemic effect of capsaicinoids by increased bile acids excretion in ovariectomized rats. Mol Nutr Food Res 57, 1080-1088.

21. Manjunatha H \& Srinivasan K (2007) Hypolipidemic and antioxidant effects of curcumin and capsaicin in high-fat-fed rats. Can J Physiol Pharmacol 85, 588-596.

22. Zhang L, Fang G, Zheng L, et al. (2013) Hypocholesterolemic effect of capsaicinoids in rats fed diets with or without cholesterol. J Agric Food Chem 61, 4287-4293.

23. Yuan LJ, Qin Y, Wang L, et al. (2016) Capsaicin-containing chili improved postprandial hyperglycemia, hyperinsulinemia, and fasting lipid disorders in women with gestational diabetes mellitus and lowered the incidence of large-for-gestationalage newborns. Clin Nutr 35, 388-393.

24. Ahuja KD \& Ball MJ (2006) Effects of daily ingestion of chilli on serum lipoprotein oxidation in adult men and women. $\mathrm{BrJ}$ Nutr 96, 239-242.
25. Ahuja KD, Robertson IK, Geraghty DP, et al. (2007) The effect of 4-week chilli supplementation on metabolic and arterial function in humans. Eur J Clin Nutr 61, 326-333.

26. Popkin BM, Du S, Zhai F, et al. (2010) Cohort Profile: The China Health and Nutrition Survey - monitoring and understanding socio-economic and health change in China, 1989-2011. Int J Epidemiol 39, 1435-1440.

27. He K, Du S, Xun P, et al. (2011) Consumption of monosodium glutamate in relation to incidence of overweight in Chinese adults: China Health and Nutrition Survey (CHNS). Am J Clin Nutr 93, 1328-1336.

28. Popkin BM (2014) Synthesis and implications: China's nutrition transition in the context of changes across other lowand middle-income countries. Obes Rev 15, Suppl. 1, 60-67.

29. Xu X, Hall J, Byles J, et al. (2015) Dietary pattern is associated with obesity in older people in China: data from China Health and Nutrition Survey (CHNS). Nutrients 7, 8170-8188.

30. Li YP, He YN, Zhai FY, et al. (2006) [Comparison of assessment of food intakes by using 3 dietary survey methods]. Zhonghua Yu Fang Yi Xue Za Zhi 40, 273-280.

31. Yang Y, Wang G \& Pan X (2002) China Food Composition 2002. Beijing: Peking University Medical Press.

32. Yang Y, Wang G \& Pan X (2005) China Food Composition 2004. Beijing: Peking University Medical Press.

33. Yao M, McCrory MA, Ma G, et al. (2003) Relative influence of diet and physical activity on body composition in urban Chinese adults. Am J Clin Nutr 77, 1409-1416.

34. Xi B, He D, Hu Y, et al. (2009) Prevalence of metabolic syndrome and its influencing factors among the Chinese adults: the China Health and Nutrition Survey in 2009. Prev Med 57, 867-871.

35. Yan S, Li J, Li S, et al. (2012) The expanding burden of cardiometabolic risk in China: the China Health and Nutrition Survey. Obes Rev 13, 810-821.

36. Zhang J, Wang Z, Wang $\mathrm{H}$, et al. (2016) Association between dietary patterns and blood lipid profiles among Chinese women. Public Health Nutr 19, 3361-3368.

37. Tudor-Locke C, Ainsworth BE, Adair LS, et al. (2003) Physical activity in Filipino youth: the Cebu Longitudinal Health and Nutrition Survey. Int J Obes Relat Metab Disord 27, 181-190.

38. Ainsworth BE, Haskell WL, Herrmann SD, et al. (2011) 2011 Compendium of Physical Activities: a second update of codes and MET values. Med Sci Sports Exerc 43, 1575-1581.

39. Zuo H, Shi Z, Yuan B, et al. (2012) Interaction between physical activity and sleep duration in relation to insulin resistance among non-diabetic Chinese adults. BMC Public Health 12, 247.

40. Siruguri V \& Bhat RV (2015) Assessing intake of spices by pattern of spice use, frequency of consumption and portion size of spices consumed from routinely prepared dishes in southern India. Nutr J 14, 7.

41. Kwon MJ, Song YS, Choi MS, et al. (2003) Red pepper attenuates cholesteryl ester transfer protein activity and atherosclerosis in cholesterol-fed rabbits. Clin Chim Acta 332, 37-44.

42. Yoshioka M, St-Pierre S, Suzuki M, et al. (1998) Effects of red pepper added to high-fat and high-carbohydrate meals on energy metabolism and substrate utilization in Japanese women. Br J Nutr 80, 503-510.

43. Kawada T, Hagihara K \& Iwai K (1986) Effects of capsaicin on lipid metabolism in rats fed a high fat diet. $J$ Nutr $\mathbf{1 1 6}$, $1272-1278$.

44. Lee GR, Shin MK, Yoon DJ, et al. (2013) Topical application of capsaicin reduces visceral adipose fat by affecting adipokine levels in high-fat diet-induced obese mice. Obesity (Silver Spring) 21, 115-122. 
45. Lee CY, Kim M, Yoon SW, et al. (2003) Short-term control of capsaicin on blood and oxidative stress of rats in vivo. Phytother Res 17, 454-458.

46. Marquez-Ibarra A, Huerta M, Villalpando-Hernandez S, et al. (2016) The effects of dietary iron and capsaicin on hemoglobin, blood glucose, insulin tolerance, cholesterol, and triglycerides, in healthy and diabetic wistar rats. PLOS ONE 11, e0152625.

47. Saito A, Nakamura K, Hori Y, et al. (2000) Effects of capsaicin on biliary free fatty acids in rats. Int J Vitam Nutr Res 70, 19-23.

48. Srinivasan K \& Sambaiah K (1991) The effect of spices on cholesterol 7 alpha-hydroxylase activity and on serum and hepatic cholesterol levels in the rat. Int J Vitam Nutr Res 61, 364-369.

49. Maeda H, Saito S, Nakamura N, et al. (2013) Paprika pigments attenuate obesity-induced inflammation in 3T3-L1 adipocytes. ISRN Inflamm 2013, 763758.

50. Medvedeva NV, Andreenkov VA, Morozkin AD, et al. (2003) [Inhibition of oxidation of human blood low density lipoproteins by carotenoids from paprika]. Biomed Khim $\mathbf{4 9}$, 191-200.

51. Aizawa K \& Inakuma T (2009) Dietary capsanthin, the main carotenoid in paprika (Capsicum annuum), alters plasma high-density lipoprotein-cholesterol levels and hepatic gene expression in rats. Br J Nutr 102, 1760-1766.

52. Zimmer AR, Leonardi B, Miron D, et al. (2012) Antioxidant and anti-inflammatory properties of Capsicum baccatum: from traditional use to scientific approach. J Ethnopharmacol 139, 228-233.

53. Jiang F \& Dusting GJ (2003) Natural phenolic compounds as cardiovascular therapeutics: potential role of their antiinflammatory effects. Curr Vasc Pharmacol 1, 135-156.

54. Kang JH, Tsuyoshi G, Le Ngoc H, et al. (2011) Dietary capsaicin attenuates metabolic dysregulation in genetically obese diabetic mice. J Med Food 14, 310-315.

55. Sun D, Lv J, Chen W, et al. (2014) Spicy food consumption is associated with adiposity measures among half a million Chinese people: the China Kadoorie Biobank study. BMC Public Health 14, 1293.

56. Sherman PW \& Hash GA (2001) Why vegetable recipes are not very spicy. Evol Hum Behav 22, 147-163.

57. Yoo S, Kim H \& Cho HI (2015) Heterogeneity in obesity status and cardiovascular risks in multiethnic Asian female immigrants in South Korea. Asia Pac J Public Health 27, NP448-NP456. 